\title{
INFORMATION RETRIEVAL EVALUATION FOR TOURISM RELATED SOCIAL MEDIA CONTENT
}

\author{
Angelina Njeguš ${ }^{1}$, \\ Verka Jovanović², \\ Marina Marjanović-Jakovljević 3
}

${ }^{1}$ Singidunum University, Faculty of Informatics and Computing

Belgrade, Serbia

${ }^{2}$ Singidunum University, Faculty of Tourism and Hospitality Management, Belgrade, Serbia

${ }^{3}$ Singidunum University, Faculty of Technical Sciences,

Belgrade, Serbia

\begin{abstract}
:
Considering that Social Media play an important role as an information source for users, many companies focus on Social Media marketing as a strategic tool for their business. According to the recent research, more then $60 \%$ of users use search engines when looking for some information. Therefore, this study aims to investigate to which extent Social Media appear in search engine results, and what is the relevance of retrieved Social Media sites. Since the goal of any Information Retrieval system is to retrieve relevant documents in response to the user's query, the set-based measure and rank-based measures are used herein. Mathematically presented, these factors are very sensitive to user relevance judgements and their properties are discussed. Through conducted experiments, Precision, Recall and Average Precision of a Google search engine are evaluated. Different tourism related topics, in terms of IR evaluation metrics, are compared. The results show that Social Media content appears in very low percentage (23.1\%) of all search results. The most visible social media are Facebook, Wikipedia and TripAdvisor.
\end{abstract}

Keywords:

social media, information retrieval, search engine, tourism.

\section{INTRODUCTION}

It is well-known that Social Media (SM) is among top three Search Engine Optimization (SEO) strategies, besides content quality and backlinks (Tober et al., 2014). From the company's perspective, it is very important that, apart from links that lead to their official web pages, also the links of their SM websites appear, so that users can raise their ratings. From user perspective, SM plays an important role as an information source (Kirchhoff et al., 2008). For example, 52\% of tourists find inspiration for travelling when using SM, where 29\% find travel planning ideas on Facebook, and 14\% are inspired by TripAdvisor's vacation planning insight (Mdg, 2012). However, according to the research of the European Tourism Association, SM is less effective when generating bookings in the short term (Rossini et al., 2014). On the other hand, according to Google research, $61 \%$ of tourists use search engines when searching for travel related information (Google, 2014), and Social Networks, such as Google + and Facebook, are ranked among the first. Therefore, this study aims to investigate to which extent SM appear in search engine results when
Correspondence:

Angelina Njeguš

e-mail:

anjegus@singidunum.ac.rs 
searching for tourism related information, and what is the relevance of the results obtained.

Another challenge is that Web 3.0 introduced smarter web based on semantic web. The idea is that data on the web are not just hyperlinked, but now there is a meaning of their interconnections through added metadata and machine interpretable statements. World Wide Web Consortium (W3C) introduced several standards that encourage webmasters to use standard data formats that will enhance semantic web content development. The Resource Description Framework (RDF) allows storing data in a standard format in order to easily identify the meaning of data when search engine processes the user query. However, smarter web is not only about semantics but also the usage of web data mining techniques in order to get knowledge about the user. The smarter web is also influenced by big data that are generated on the web every second. Therefore, there is a need for tools that will process and analyze big data. In practice, all of these mean, that user, through advanced search engine, can ask the specific question (search query) and receive almost concrete answers (relevant information). However, a huge effort is in front of the search engines who seek to improve relevance, and importance of search results by understanding user intention and the contextual meaning of user's query (Girit et al., 2012).

Most search engines use search methods that are not convenient for indexing and retrieval of semantic markup content (Finin et al., 2005). When detecting embedded markup within a document, search engines index it, but not process the markup. Unfortunately, the semantic markups (such as RDF or OWL) are typically used as knowledge representation languages rather than markup. Therefore, the most search engines today cannot use semantic markup to improve information retrieval. However, Google has several ongoing projects and patents in the field of semantic search, such as: Assigning terms of interest to an entity; Self learning semantic search engine; Knowledge graph based search system and other. In order to find out to what extent the information retrieval systems of today's semantic search engines are improved, in this paper, their precision, average precision and recall of information retrieval system using keywords to complete phrases of tourism related topics are done.

This study is organized as follows: the first section provides the background of the research, then the Information Retrieval System Model is given, and the properties of evaluation metrics are discussed, and at the end the experiment design and results are analyzed.

\section{RESEARCH BACKGROUND}

Development of dynamic websites was the trigger for switching from static Web 1.0 to a dynamic and highly social Web 2.0. Soon after, websites that enable users to communicate with each other, share pictures, videos, reviews, customize personal profiles, write blogs and other, were developed. These websites are called SM websites. However, the slight revolution in people's social life has brought the advent of Social Networks. In favour of this fact are the figures of social network users where it is estimated that there are around 2.13 billion users (Statista, 2015).

The SM refers to a group of dynamic web-based applications where its content is mostly generated by the users, so called user-generated content (UGC) sites (Kaplan et al., 2010). The current examples of SM websites include social networking sites (e.g. Facebook, LinkedIn, Twitter), Social News, Photo Sharing (e.g. Instagram, Pinterest), Video Sharing sites (e.g. YouTube), Online communities, Blogs, Forums, FAQ, Commenting, Traditional news, Virtual worlds etc. SM has a social structure made of many connected nodes (individuals or objects), that are composed of the concept and role in a form of sub-object that has various characteristics. Nodes are direct/indirect connected by specific types (such as friendship, dislike, financial exchange). The overall graph model of SM forms the entire network (Choi et al., 2012).

In recent studies, it has been argued that SM started replacing traditional information sources, and later then that SM influence the users' online purchasing behavior process (Vinerean et al., 2013; Ioanas et al., 2014). Since SM has a significant impact on user' information acquisition, much effort is done in the field of application that analyse the content of Social Network and information retrieval systems (Kirchhoff et al., 2008; Nasution et al., 2012; Korfiatis et al., 2008). With advancement of the web, major search engines constantly introduce new features. However, most information retrieval systems have limited abilities to give personalized response to the user request (Fernandez et al., 2011; Zidi et al., 2013). Higher-level natural language processing techniques such as chunking, word sense disambiguation, parsing, compound recognition, part-of-speech tagging and other methods, used in information retrieval, have recorded very slow improvements (Brants, 2003; Ferrandez et al., 2013).

Information retrieval (IR), as part of computer science, studies the effective and efficient retrieval of relevant information from a collection of web documents in order to satisfy a user information need (Mehlitz et al., 
2007; Crestani et al., 2001). There are several ongoing issues such as relevance, performance, scalability, and other. Relevancy from user perspective is subjective (user's judgment), situational (depends on user's needs), cognitive (depends on user perception), and dynamic (changes over time) (Borlund, 2003). Another problem is the size of the Web, where it is estimated that it contains at least 4.72 billion pages (www.worldwidewebsize.com, from November, 2015).

Evaluation of search engine systems is highly important for measuring how successfully an IR system fulfills users' information needs (Clough et al., 2013). At the beginning, this evaluation was mostly system-oriented i.e. conducted in a laboratory setting environment with the main focus on search algorithms, disk space, system speed, and other relevant system factors, but with no influence or with very little involvement of end users. Today, it is mostly user-oriented which takes into account the user's context, interactions with the system, information searching behavior, satisfaction with the search results and others (Borlund, 2009; Maskari et al., 2011). Therefore, more research is dealing with interactive information retrieval (IIR) evaluation (Kelly, 2009). An important role in establishing standards for IIR evaluation belongs to the work of the organization of the information retrieval evaluation events - Text REtrieval Conference (TREC) Interactive Track (Figure 1).

IR system evaluation is usually test collection based. The test collection contains a set of statements expressed as keywords, questions or longer written descriptions that are related to some topic. In this paper, tourism related topics are used. The selection of keywords is done upon a number of prevoius studies and recent trends in tourism. The most searched keywords, that reflect the broad tourism topics, are "hotels", "restaurants", "wellness", "nightlife", and other (Xiang et al., 2010). Recent development in tourism involve Medical tourism, Educational tourism, Sports tourism and other. In order to be consistent with the information search context, the focus of the study is on the capital city Belgrade, as an urban destination. Therefore, the selected pre-defined keywords are combined with destination name to form queries, which were entered then into Google.

In order to measure IR effectiveness, there are setbased evaluation measures (precision and recall), rankbased measures, and other. To evaluate the performance of IR system, the paper measures how far down the ranked list of results will a user need to look to find SM relevant documents. The objective of the experiment is to evaluate precision, average precision and recall of a Google search engine.

According to the statistics, Google is the most used search engine in the world, with 1.1 billion of unique monthly visitors (eBiz, 2015), and $67.5 \%$ of market share. At the beginning of the search engine era, Google ranked pages according to the number of frequently used keywords. Today, the goal of semantic search created the set of new criteria such as the number of backlinks, user signals etc. Due to this development, ranking factors are constantly evolving. According to the Google rankingfactors 2014 (Tober, 2014), the social networks, such as Google +, and Facebook, are among the first. However, the herein presented experiment demonstrates that Google does not rank, or in very low percentage shows the SM as search results.

\section{INFORMATION RETRIEVAL SYSTEM MODEL}

\section{System model flowchart}

In this paper adjusted system model flowchart is used (Marjanovic-Jakovljevic, 2015a) (Figure 2). On the left side of the diagram, shown in Figure 2, a user query is presented. In this paper, tourism related topics are used. In that stage, the user can enter some query, like "Visit Belgrade" or "Book a hotel in Belgrade" etc.

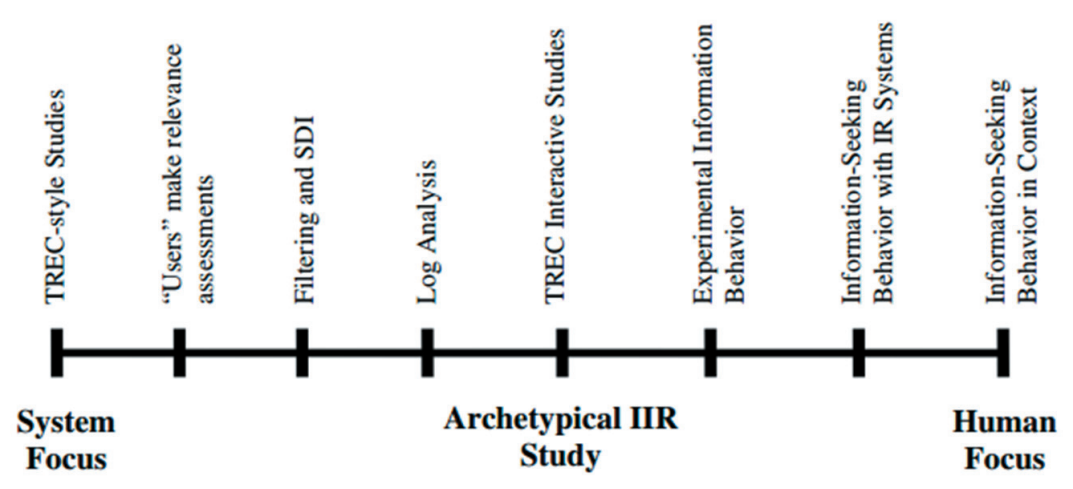

Figure 1. The history of IR system studies Source: Kelly (2009). 
On the other hand, web provides huge amount of ecommerce market data provided by tourism companies, such as hotels, restaurants, online travel agencies. Search engine system includes both documents, and estimates at what degree ranked web documents meet user's information requirements. The user rates retrieved documents as relevant or non-relevant. Therefore, the relevance of the document is assessed according to the user information need, not on query. The challenge in any IR system is to retrieve only the desired web documents, so various system performance criteria have been used to measure the IR system effectiveness. In this paper, Google search engine is used for measuring the IR system effectiveness.

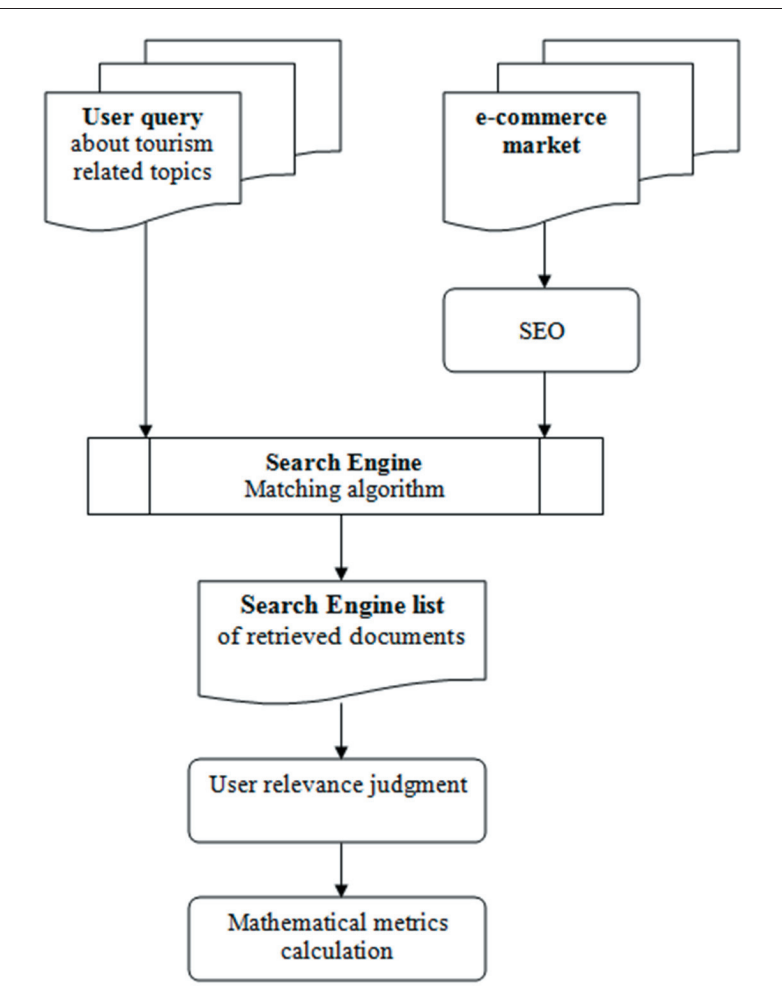

Figure 2. System Model Flowchart

\section{Search Engine evaluation metrics properties}

There are several evaluation measures in order to assess the system effectiveness. The two most frequent are: set-based measures (Precision and Recall), and rank-based measures (Average Precision). Those measures are applied to the pages links from the ranking list, assuming that the relevance judgment is known. In this paper, binary relevance (relevant or non-relevant) is assumed rather than graded relevance (relevant, partially-relevant, or non-relevant) (Kekalainen, 2005). In (Zhu, 2004), evaluation of those parameters is explained and some of their properties are already discussed in (Marjanovic-Jakovljevic, 2015).
Precision is calculated as the ratio of the number of relevant retrieved web documents $\left(N_{\text {rel_ret }}\right)$, and the number of retrieved web documents within the ranking list $\left(N_{r e t}\right)$. In other words, precision measures the capability of the IR system to retrieve only relevant web documents. However, it is influenced by retrieval of non-relevant web documents.

$$
\text { Precision }=\frac{N_{\text {rel_ret }}}{N_{\text {ret }}}
$$

Recall measures the ability to find all relevant web documents within the entire result list, which is, in the case of web search, a time-consuming job. It is defined as:

$$
\text { Recall }=\frac{N_{\text {rel_ret }}}{N_{\text {rel }}}
$$

Where $N_{\text {rel }}$ presents the number of relevant web documents within the entire result list.

Average Precision (Buckley et al., 2000) measures ranked retrieval results, with focus on returning maximum number of relevant documents that are positioned higher in the ranked list. In other words, Average Precision is the mean of the Precision that is calculated after retrieval of each relevant document. If the observed document is assumed as non relevant, Precision has a value of zero. Therefore, Average Precision might be expressed as:

AveragePrecision $(\mathrm{i})=\frac{1}{N_{\text {rel_ret }}} \sum_{i=1}^{N_{\text {ret }}} x_{i} \operatorname{Precision}_{i}=\frac{1}{N_{\text {rel_ret }}} \sum_{i=1}^{N_{\text {ret }}} \frac{x_{i}}{i} \sum_{k=1}^{i} x_{k}$

where:

$$
x_{k}=\left\{\begin{array}{l}
1 \text { if } k^{\text {th }} \text { page link is relevant } \\
0 \text { if } k^{\text {th }} \text { page link is not relevant }
\end{array}\right.
$$

All relevant documents that are retrieved may or may not belong to SM. To estimate the significance of SM marketing, the probability of SM in the Google ranked list is calculated such as:

$$
P=\frac{N_{\text {appearings }}}{N_{\text {rel_ret }}}
$$

where $N_{\text {appearings }}$ presents the number of relevant SM sites within a ranked list.

According to (1), after $N_{\text {ret }}$ ranked retrieved documents, the Precision is calculated as (6):

$$
\text { Precision }_{N}=\frac{1}{N_{\text {ret }}} \sum_{k=1}^{N_{\text {ret }}} x_{k}
$$


From (2), using the same notations, Recall can be calculated such as (7):

$$
\text { Recall }_{k}=\frac{1}{N_{\text {rel }}} \sum_{k=1}^{N_{\text {ret }}} x_{k}
$$

From (7), it is obvious that Average Precision is influenced by the number of retrieved relevant documents and their position within the ranked list. For the sake of illustration, in Fig. 3 we assume that we have only one relevant page link on the $i^{\text {th }}$ position within the rank list, where $1 \leq i \leq N_{\text {ret }}$. In such case, Average Precision parameter is calculated as:

$$
\text { AveragePrecision }(i)=\frac{1}{i}
$$

and Recall parameter has the constant value:

$$
\text { Recall }=\frac{1}{N_{\text {rel }}}=\text { const. }
$$

The difference of Average Precision (Fig. 3b) is calculated as:

$$
\Delta \text { AveragePrecision }(i)=-\frac{1}{i \cdot(i+1)}
$$

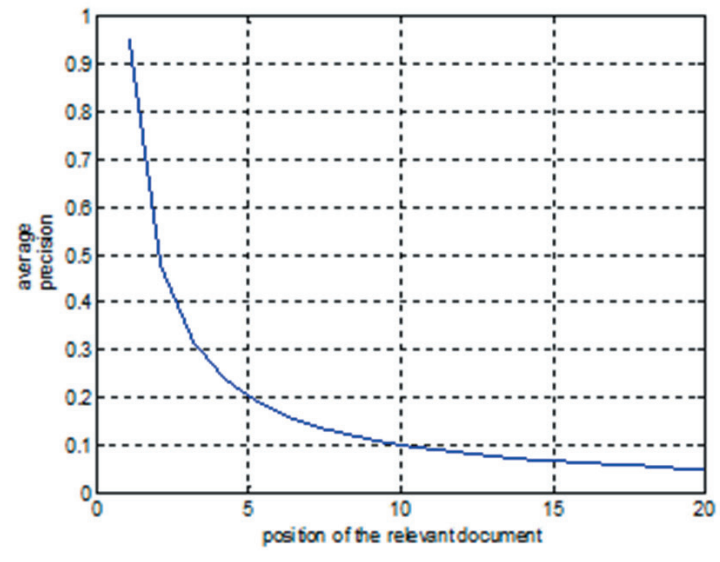

(a) Average Precision vs. Position of the relevant page link: Nrel_ret=1;

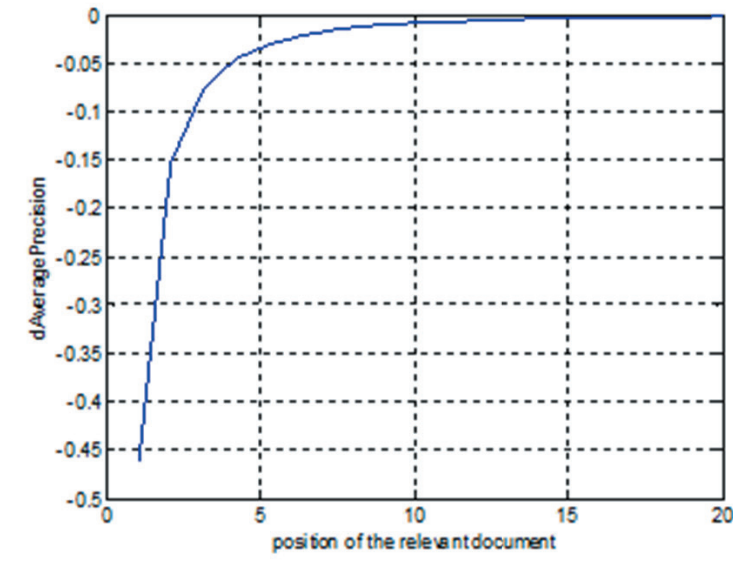

(b) $\Delta$ Average Precision vs. Position of the relevant page link: Nrel_ret=1;

Figure 3. shows that the value of Average Precision and the difference of Average Precision is particularly expressed from $1^{\text {st }}$ to $10^{\text {th }}$ position, when having relevant document on two adjacent positions.

Since the relevance judgment depends only on user preferences, it will be interesting to examine what is the regularity of the change in the Average Precision, if switching a number of non-relevant into relevant. Supposing that $k_{\text {switched }}$ presents the number of the switched judgments, we have $2^{k_{\text {swithed }}}\left(\begin{array}{l}N_{\text {ret. }} \\ k_{\text {switched }}\end{array}\right)$ different possibilities.

First, we assume only one switched judgment, i.e. $k_{\text {switched }}=1$ on the $j^{\text {th }}$ position, where $1 \leq j \leq N_{\text {ret }}$. Therefore, the obtained difference of Average Precision is expressed as (11):

$\Delta$ AveragePrecision $(j)=\operatorname{AveragePrecision}\left(x_{j}=1\right)-\operatorname{AveragePrecision}\left(x_{j}=0\right)$

Average Precision in case where $j^{\text {th }}$ page link is not relevant, is presented as (12):

$$
\operatorname{AveragePrecision}\left(x_{j}=0\right)=\frac{1}{N_{\text {rel_ret }}}\left(\sum_{i=1}^{j-1} x_{i} \text { Precision }_{i}+\sum_{i=j+1}^{N_{\text {ret }}} x_{i} \text { Precision }_{i}\right)
$$


While, in the case where $j^{\text {th }}$ document is relevant, the Average Precision is presented as (13):

$$
\operatorname{AveragePrecision}\left(x_{j}=1\right)=\frac{1}{\left(N_{\text {rel_ret }}+1\right)}\left(\sum_{i=1}^{j-1} x_{i} \text { Precision }_{i}+\text { Precision }_{j}+\sum_{i=j+1}^{N_{r e t}} x_{i}^{\prime} \text { Precision }_{i}^{\prime}\right)
$$

$x_{i}^{\prime}$ present relevance judgments from $j^{\text {th }}$ position and it is considered that $x_{i}^{\prime}=x_{i}$ for $1 \leq i^{\prime}=i \leq N_{\text {ret. }}$ and $N_{\text {rel_ret }}>1$, therefore, the change of Average Precision is calculated as:

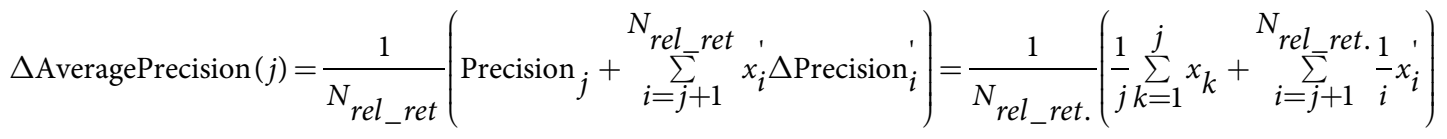

where $\Delta$ Precision $_{i}^{\prime}=\frac{1}{i}$ presents the Precision difference on the $i^{\text {th }}$ position where $x_{i}^{\prime}=1$.

Given that $k$ simultaneous switches in relevance judgment produce the same difference of Average Precision as $k$ successive single switches, therefore the total change of Average Precision can be expressed as a sum of Average Precision differences. Since $\Delta$ AveragePrecision $(j)>\Delta$ AveragePrecision $(j+1)$, it is clear that the value of $\Delta$ AveragePrecision is higher when the switch is performed on the top of the ranked list.

\section{EXPERIMENT DESIGN AND RESULTS}

In this study, the following parameters have to be specified: search engine, search queries, data processing, and analysis. Since Google is the most used search engine (Malik, 2014), in this experiment the precision of Google search engine has been evaluated. Generally, Google handles three major types of user queries, such as navigational, informational and transactional queries (Enge et al., 2012). Navigational queries mean that the user knows what is looking for, so it is enough to type "YouTube", and Google recognizes what the user wants, and shows the desired page. Informational queries mean that the user types several keywords, such as "bike", "tourism" etc, and Google are using its relevancy algorithm with semantic features to display search results. Transactional queries are used when the user wants to purchase something on the web, and types for example "book a hotel", or "I want to do aesthetic surgery in Belgrade". Here the Google's semantic function plays an important role.

In this experiment, semantic search queries are formulated using natural language. Acting as a foreign tourist who wants to visit Belgrade, questions are placed in the English language. For queries, a wide range of tourism related topics, such as medical tourism, educational tourism, were used. To research the relevance of search results, all three IR evaluation parameters are calculated. In addition, the type and position of the SM of a relevant page, within the Google ranked list, is observed. In this experiment "hands-on" research is done. In order to make judgment about the relevance of web pages, the following is considered:
- the web page is considered relevant if it is closely matched to the subject matter of the search query, and

- the page is categorized as non-relevant if it consists of a whole series of links, or the site can not be accessed, or is not related to the subject matter.

The column Relevance judgments, presented in the Tables 1-4, shows the position of relevant (signed with "1") or non-relevant (signed with " 0 ") document within a result of 20 ranked web documents. Observing all three parameters, with the goal to present the significance of SM marketing, three experiments are described.

In the first experiment, different narrow related tourism topics, in term of IR evaluation parameters, are compared. The queries are: "visit Belgrade", "hotels in Belgrade", "restaurants in Belgrade", "wellness in Belgrade" and etc. The highest accuracy is achieved when searching hotels. The average highest precision (means when the document is listed at the beginning more times than others) is obtained when searching restaurants. The most appearing SM sites are TripAdvisor followed by Facebook.

In the second experiment, different health topics, as wider tourism related topics, in the term of IR evaluation parameters, are compared. The queries are: "private clinics in Belgrade", "dental implant clinics in Belgrade", "aesthetic clinics in Belgrade", "acupuncture in Belgrade" etc. The biggest average precision of $100 \%$ was achieved when searching aesthetic clinic in Belgrade, since the top ten positions are relevant pages. The worst results, according to all three parameters, are obtained when searching private clinics in Belgrade. SM appear in a very low percentage (Table 2 ).

In the third experiment, different educational tour- 
Table 1. IR metrics of narrow tourism related topics

\begin{tabular}{|c|c|c|c|c|c|c|}
\hline Query & $\begin{array}{l}\text { Relevance } \\
\text { judgments }\end{array}$ & Avg. Precision & Precision & Recall & $\begin{array}{l}\text { Social Media } \\
\text { (followed by its } \\
\text { position) }\end{array}$ & $P$ \\
\hline 1 & $\begin{array}{l}11010 \\
00101 \\
00110 \\
00000\end{array}$ & 0.47 & 0.35 & 0.7 & $\begin{array}{l}\text { 2. Facebook } \\
\text { 4. TripAdvisor }\end{array}$ & 0.285 \\
\hline 2 & $\begin{array}{l}11110 \\
01110 \\
01010 \\
10000 \\
\end{array}$ & 0.5 & 0.82 & 1 & $\begin{array}{l}\text { 2. TripAdvisor } \\
\text { 16. TripAdvisor }\end{array}$ & 0.200 \\
\hline 3 & $\begin{array}{l}11111 \\
00010 \\
01000 \\
00000\end{array}$ & 0.62 & 0.35 & 0.7 & $\begin{array}{l}\text { 1. TripAdvisor } \\
\text { 12. TripAdvisor }\end{array}$ & 0.285 \\
\hline 4 & $\begin{array}{l}11001 \\
00000 \\
01010 \\
00000 \\
\end{array}$ & 0.35 & 0.25 & 0.5 & 13. Facebook & 0.200 \\
\hline \multicolumn{6}{|c|}{ Average $P$} & 0.243 \\
\hline
\end{tabular}

Table 2. IR metrics of health tourism topics

\begin{tabular}{|c|c|c|c|c|c|c|}
\hline Query & $\begin{array}{l}\text { Relevance } \\
\text { judgments }\end{array}$ & Avg. Precision & Precision & Recall & Social Media & $P$ \\
\hline 1 & $\begin{array}{l}01101 \\
10101 \\
00010 \\
00000\end{array}$ & 0.42 & 0.35 & 0.7 & 2. Blogspot & 0.285 \\
\hline 2 & $\begin{array}{l}11110 \\
11111 \\
00100 \\
00000\end{array}$ & 0.91 & 0.5 & 1 & 10. Youtube & 0.100 \\
\hline 3 & $\begin{array}{l}11111 \\
11111 \\
00000 \\
00000\end{array}$ & 1 & 0.5 & 1 & & 0 \\
\hline 4 & $\begin{array}{l}11111 \\
11101 \\
00010 \\
00000\end{array}$ & 0.96 & 0.5 & 1 & & 0 \\
\hline \multicolumn{6}{|c|}{ Average $P$} & 0.195 \\
\hline
\end{tabular}

Table 3. IR metrics of educational tourism topics

\begin{tabular}{|c|c|c|c|c|c|c|}
\hline Query & $\begin{array}{l}\text { Relevance } \\
\text { judgments }\end{array}$ & Avg. Precision & Precision & Recall & Social Media & $P$ \\
\hline 1 & $\begin{array}{l}11011 \\
11100 \\
00010 \\
10010 \\
\end{array}$ & 0.78 & 0.5 & 1 & 1. Wikipedia & 0.100 \\
\hline 2 & $\begin{array}{l}11101 \\
01001 \\
00010 \\
01010 \\
\end{array}$ & 0.66 & 0.45 & 0.9 & $\begin{array}{l}\text { 2. Wikipedia } \\
\text { 3. Wikipedia } \\
\text { 7. Facebook }\end{array}$ & 0.333 \\
\hline 3 & $\begin{array}{l}11111 \\
11100 \\
01100 \\
00000 \\
\end{array}$ & 0.94 & 0.5 & 1 & $\begin{array}{l}\text { 4. Wikipedia } \\
\text { 6. LinkendIn } \\
\text { 7. Facebook } \\
\text { 8. Facebook }\end{array}$ & 0.400 \\
\hline 4 & $\begin{array}{l}10011 \\
00000 \\
01010 \\
00000 \\
\end{array}$ & 0.28 & 0.25 & 0.5 & 1. Wikipedia & 0.200 \\
\hline \multicolumn{6}{|c|}{ Average $\mathrm{P}$} & 0.250 \\
\hline
\end{tabular}


ism topics, in terms of IR evaluation parameters are compared. The queries are: "private universities in Belgrade", "public universities in Belgrade", "college of electrical engineering in Belgrade", "college of software engineering in Belgrade" etc. The best results, in terms of average precision, are achieved during the search of electrical engineering in Belgrade, which also appears mostly on SM, such as Wikipedia, LinkedIn and Facebook. While the worst results, in terms of all parameters, were achieved when searching the college of software engineering. Generally, it can be concluded that most universities are promoting via SM, and the most on Facebook, LinkedIn, and Wikipedia.

\section{CONCLUSION}

This study firstly confirms the growing significance of SM as a valuable information source from user's aspect, and strategic marketing tool from the company's perspective. Today, more users, before making a final decision, want to read opinions of others about the specific product or service. An increasing number of available SM allows them to do so. Since the efficiency of information retrieval systems is measured by how quickly relevant documents are returned and presented on the first page, and how well it reflects user's needs, the question that arises is to what extent IR systems represent SM content in search results. Topics that are used in conducted experiment are related to different types of tourism as they are among the most searched content on the Internet. In order to evaluate the quality of IR systems, whose development today is influenced by the occurrence of Semantic content on the Web, three main parameters were observed (Precision, Recall, and Average Precision).

Mathematically, it has been shown that these factors are influenced by users' judgment which mostly depends on user preferences. Based on the mathematical model, it is demonstrated that the Average Precision decreases with decreasing position of the relevant document. This difference in Average Precision is particularly expressed from 1st to 10th position of relevant documents in the Google ranking list. Since this mostly depends on the user judgment, it is examined the regularity of the change in the Average Precision, replacing a number of non-relevant into relevant documents. This fact significantly simplifies the analysis of user judgment influence on Average Precision, which is very important parameter in IR system evaluation.

Empirically, it is shown that, depending on the topic, different SM content is represented. However, the SM content appear in a very low percentage $(23.1 \%)$ of all search results. The most visible SM are Facebook, Wikipedia, and TripAdvisor. There may be numerous reasons as to why SM do not occur in a large number. For instance, it could be that Facebook is blocking access to the crawlers so as not to burden their servers, or that tourism content is just not sufficiently represented in $\mathrm{SM}$, or is not written in the English language.

\section{REFERENCES}

Amerland, D. (2014). Google ${ }^{T M}$ Semantic Search: Search Engine Optimization (SEO) Techniques That Get Your Company More Traffic, Increase Brand Impact, and Amplify Your Online Presence. Indianapolis: Pearson Education, Inc.

Borlund, P. (2003). The concept of relevance in IR. Journal of the American Society for Information Science and Technology, 54(10), 913-925.

Borlund, P. (2009). User-centered evaluation of information retrieval systems. In A. Göker and J. Davies (Eds.), Information retrieval: searching in the 21st Century. Chicester, UK: John Wiley \& Sons.

Brants, T. (2003). Natural Language Processing in Information Retrieval. In Proc. of the 14th meeting of computational linguistics in Netherlands (CLIN 2003).

Buckley, C., \& Vorhees, E.M. (2000). Evaluating evaluation measure stability. In Proc. of the 23rd Annual International ACM SIGIR Conference on Research and Development in Information Retrieval, Ed. (pp. 33-40).

Clough, P., \& Sanderson, M. (2013). Evaluating the performance of information retrieval systems using test collections. Information research, 18(2), 1-16.

Crestani, F., \& Lalmas, M. (2001). Logic and Uncertainty in Information Retrieval. Lectures on Information Retrieval. Springer-Verlag Berlin Heidelberg (pp. 179-206).

eBiz (2015). Top 15 Most Popular Search Engines - October 2015. eBizMBA. Retrived August 5, 2016 from http://www.ebizmba.com/articles/search-engines.

Enge, E., Spencer, S., Stricchiola, J., \& Fishkin, R. (2012). The Art of SEO. (2nd ed.). Sebastopol, CA: O'Reilly Media.

Fernandez, M., Cantador, I., Lopez, V., Vallet, D., Castells, P., \& Motta, E. (2011). Semantically enhanced Information Retrieval: An ontology-based approach. Web Semantics: Science, Services and Agents on the World Wide Web, 9(4), 434-452. doi:10.1016/j.websem.2010.11.003

Ferrandez, A., Rojas, Y., \& Peral, J. (2013). The successful application of natural language processing for information retrieval. Journal of Computer Science and Technology, 7(1), 79-85. 
Finin, T., Mayfield, J., Joshi, A., Scott Cost, R., \& Fink, C. (2005). Information Retrieval and the Semantic Web. In Proc. of the 38th Annual Hawaii International Conference on System Sciences (HICSS'05).

Girit, H., Eberhard, R., Michelberger, B., \& Mutschler, B. (2012). On the Precision of Search Engines: Results from a Controlled Experiment. In Proc. of 15th International Conference, BIS 2012, Vilnius, Lithuania, May 21-23, 2012 (pp. 201-212).

Google (2014). The 2014 Traveler's Road to Decision. Retrived August 11, 2016 from https://think.storage. googleapis.com/docs/2014-travelers-road-to-decision_research_studies.pdf

Ioanas, E., \& Stoica, I. (2014). Social Media and its Impact on Consumer Behavior. International Journal of Economic Practices and Theories, 4(2), 295-303.

Kaplan, A.M., \& Haenlein, M. (2010). Users of the world, unite! The challenges and opportunities of Social Media. Business Horizons, 53(1), 59-68. doi: 10.1016/j.bushor.2009.09.003

Kekalainen, J. (2005). Binary and graded relevance in IR evaluations - Comparison of the effects on ranking of IR systems. Information Processing and Management, 41, 1019-1033.

Kelly, D. (2009). Methods for evaluating interactive information retrieval systems with users. Foundations and Trends in Information Retrieval, 3(1-2), 1-224.

Kirchhoff, L., Stanoevska-Slabeva, K., Nicolai, T., \& Fleck, M. (2008). Using Social Network analysis to enhance information retrieval systems. Applications of Social Network Analysis, 7, 1-21.

Korfiatis, N., Sicilia, M-A., Hess, C., Stein, K., \& Schlieder, C. (2007). Social Network Models for Enhancing Reference - Based Search Engine Rankings. In Social information retrieval systems: emerging technologies and applications for searching the web effectively, D. Goh and S. Foo, Eds., IGI Global (pp. 109-133).

Lee, J., Stern, T.I., Donaker, G.J., \& Blair-Goldensohn S.J. (2013). Assigning terms of interest to an entity. United States Patent. Google Inc.

Malik S. (2014). A Comparative Study of two major Search Engines: Google and Yahoo. Oriental journal of computer science and technology, 7(1).

Marjanovic-Jakovljevic, M. (2015a). Information Retrieval System for Serbian Language: Requirements, Limitations, and Experiments. International Journal of Computer Applications, 5(5), 22-34.

Marjanovic-Jakovljevic, M. (2015b). Sensitivity Analysis of Information Retrieval Metrics. International Journal of Computer Science \& Information Technology (IJCSIT), 7(4), 45-56.

Maskari A., \& Sanderson, M. (2011). The effect of user characteristics on search effectiveness in informati- on retrieval. Journal of Information Processing and Management, 47( 5), 719-729.

MDG. (2012). Vacationing the Social Media Way (Infographic). MDG Advertising. Retrived August 5, 2016 from www.mdgadvertising.com/blog/vacationing-the-social-media-way-infographic/.

Mehlitz, M., Bauckhage, C., Kunegis, J., Albayrak, S. (2007). A New Evaluation Measure for Information Retrieval Systems. IEEE International Conference on Systems, Man and Cybernetics. Montreal, Que. (pp. 1200-1204).

Nasution, M.K., Noah, S.A. (2012). Information Retrieval Model: A Social Network Extraction Perspective. In Proc. of 2012 International Conference on Information Retrieval and Knowledge Management, CAMP'12. 13-15 March 2012. Kuala Lumpur, Malaysia.

Okkyung, C., Kangseok, K., Duskang, W., Hongjin, Y., \& Manpyo, H. (2012). Personalized mobile information retrieval system. International Journal of Advanced Robotic Systems, 9, 1-7.

Rossini, A., Reed, R., \& Timmons, J. (2014). The new online travel consumer. Euromonitor International. Retrived August 10, 2016 from http://www. etoa.org/docs/default-source/presentations/2014the-new-online-travel-consumer.pdf

Saracevic, T. (1995). Evaluation of evaluation in information retrieval. In Edward A. Fox, Peter Ingwersen, Raya Fidel (Eds.). In Proc. of 18th Annual International ACM SIGIR Conference on Research and Development in Information Retrieval, Seattle, Washington, USA.

Statista. (2015). Leading Social Networks worldwide as of August 2015, ranked by number of active users (in millions). The Statistics Portal. Retrived August 7, 2016 from http://www.statista.com/statistics/272014/ global-social-networks-ranked-by-number-of-users

Tober, M., Henning, L., \& Furch, D. (2014). SEO Ranking Factors and Rank Correlations 2014 - Google U.S. Whitepaper. Searchmetrics.

Vinerean, S., Cetina, I., Dumitrescu, L., \& Tichindelean, M. (2013). The Effects of Social Media Marketing on Online Consumer Behavior. International Journal of Business and Management, 8(14), 66-79.

Xiang, Z., \& Gretzel, U. (2010). Role of Social Media in online travel information search. Tourism Management, 31(2), 179-188.

Zhu, M. (2004). Recall, Precision and Average Precision. Working Paper 2004-09. Department of Statistics and Actuarial Science, University of Waterloo.

Zidi, A., \& Abed, M. (2013). A Generalized Framework for Ontology-Based Information Retrieval. International Conference on Advanced Logistics and Transport (ICALT). IEEE, Sousse, 29-31 May 2013 (pp. 165-169). 